\title{
Multiplication Operator with BMO Symbols and Berezin Transform
}

\section{Xue Feng, Kan Zhang, Jianguo Dong, Xianmin Liu, and Chi Guan}

College of Sciences, Shenyang Agricultural University, Shenyang 110866, China

Correspondence should be addressed to Kan Zhang; kanzhang2004@163.com

Received 26 May 2014; Accepted 16 November 2014

Academic Editor: Janusz Matkowski

Copyright (C) 2015 Xue Feng et al. This is an open access article distributed under the Creative Commons Attribution License, which permits unrestricted use, distribution, and reproduction in any medium, provided the original work is properly cited.

We discuss multiplication operator with a special symbol on the weighted Bergman space of the unit ball. We give the necessary and sufficient conditions for the compactness of multiplication operator on the weighted Bergman space of the unit ball.

\section{Introduction}

Let $B_{n}$ denote the unit ball in $\mathbb{C}^{n}$, and let $v$ be the normalized Lebesgue volume measure on $B_{n}$. For $-1<\alpha<\infty$, we denote by $v_{\alpha}$ the measure on $B_{n}$ defined by $d v_{\alpha}(z)=c_{\alpha}(1-$ $\left.|z|^{2}\right)^{\alpha} d v(z)$, where $c_{\alpha}=\Gamma(n+\alpha+1) / n ! \Gamma(\alpha+1)$ is a normalizing constant such that $v_{\alpha}\left(B_{n}\right)=1$. For $1 \leq p<\infty$, we write $\|\cdot\|_{\alpha, p}$ for the norm on $L^{p}\left(B_{n}, d v_{\alpha}\right)$ and $\langle\cdot, \cdot\rangle_{\alpha}$ for the inner product on $L^{2}\left(B_{n}, d v_{\alpha}\right)$. The Bergman space $A_{\alpha}^{2}\left(B_{n}\right)$ is the space of holomorphic functions which are square-integrable with respect to measure $d v_{\alpha}$ on $B_{n}$. Reproducing kernels $K_{w}^{\alpha}$ and normalized reproducing kernels $k_{w}^{\alpha}$ in $A_{\alpha}^{2}\left(B_{n}\right)$ are given by

$$
\begin{gathered}
K_{w}^{\alpha}(z)=\frac{1}{(1-\langle z, w\rangle)^{n+\alpha+1}}, \\
k_{w}^{\alpha}(z)=\frac{\left(1-|w|^{2}\right)^{(n+\alpha+1) / 2}}{(1-\langle z, w\rangle)^{n+\alpha+1}},
\end{gathered}
$$

respectively, for $z, w \in B_{n}$. For every $h \in A_{\alpha}^{2}\left(B_{n}\right)$ we have $\left\langle h, K_{w}^{\alpha}\right\rangle_{\alpha}=h(w)$, for all $w \in B_{n}$. The orthogonal projection $P_{\alpha}$ of $L^{2}\left(B_{n}, d v_{\alpha}\right)$ onto $A_{\alpha}^{2}\left(B_{n}\right)$ is given by

$$
\left(P_{\alpha} g\right)(w)=\left\langle g, K_{w}^{\alpha}\right\rangle_{\alpha}=\int_{B_{n}} g(z) \frac{1}{(1-\langle w, z\rangle)^{n+\alpha+1}} d v_{\alpha}(z),
$$

for $g \in L^{2}\left(B_{n}, d v_{\alpha}\right)$ and $w \in B_{n}$.
Given $f \in L^{1}\left(B_{n}, d v_{\alpha}\right)$, the Toeplitz operator $T_{f}: L^{2}\left(B_{n}\right.$, $\left.d v_{\alpha}\right) \rightarrow A_{\alpha}^{2}\left(B_{n}\right)$, the Hankel operator $H_{f}: L^{2}\left(B_{n}, d v_{\alpha}\right) \rightarrow$ $\left(A_{\alpha}^{2}\left(B_{n}\right)\right)^{\perp}$, and the multiplication operator $M_{f}: A_{\alpha}^{2}\left(B_{n}\right) \rightarrow$ $L^{2}\left(B_{n}, d v_{\alpha}\right)$ are given by

$$
\begin{gathered}
\left(T_{f} h\right)(z)=\int_{B_{n}} \frac{f(\omega) h(\omega)}{(1-\langle z, \omega\rangle)^{n+\alpha+1}} d v_{\alpha}(\omega), \\
\left(H_{f} h\right)(z)=f(z) g(z)-\left(T_{f} g\right)(z), \\
M_{f}(h)=f h,
\end{gathered}
$$

respectively. For $f \in L^{1}\left(B_{n}, d v_{\alpha}\right)$, we define the Berezin transform of $f$ to be the function $\tilde{f}$; that is,

$$
\widetilde{f}(z)=\int_{B_{n}} f(w)\left|k_{z}^{\alpha}(w)\right|^{2} d v_{\alpha}(w)
$$

If $f$ is bounded, then $\widetilde{f}$ is a bounded function on $B_{n}$. Since the kernels $k_{z}^{\alpha}$ converge weakly to zero as $z$ tends $\partial B_{n}$, we have that if $f$ is compact, then $\tilde{f} \rightarrow 0$ as $z \rightarrow \partial B_{n}$. The converse (in both cases) is not necessarily true. According to the definition of Berezin transform, the mean oscillation of $f$ in the Bergman metric is the function $\operatorname{MO}(f)(z)$ defined on $B_{n}$ by

$$
\operatorname{MO}(f)(z)=\widetilde{|f|^{2}}(z)-|\tilde{f}(z)|^{2}
$$


For $z \in B_{n}$, let $\psi_{z}$ be the automorphism of $B_{n}$ such that $\psi_{z}(0)=z$ and $\psi_{z}=\left(\psi_{z}\right)^{-1}$. Thus, we have the change-ofvariable formula

$$
\int_{B_{n}} h\left(\psi_{z}(w)\right)\left|k_{z}^{\alpha}(w)^{2}\right| d v_{\alpha}(w)=\int_{B_{n}} h(w) d v_{\alpha}(w),
$$

for every $h \in L^{1}\left(B_{n}, d v_{\alpha}\right)$.

Multiplication operators are one of the most widely studied classes of concrete operators. The study of their behavior on the Hardy and Bergman spaces has generated an extensive list of results in the operator theory and in the theory of function spaces [1-6]. One of the useful approaches is the use of the Berezin transform [7-11]. This method is motivated by its connections with quantum physics and noncommutative geometry.

In general, Berezin transform $\tilde{f}$ plays important role in giving necessary and sufficient conditions for the boundedness and compactness of the Toeplitz operator [12, 13]. However Berezin transform $\widetilde{|f|^{2}}$ or the mean oscillation $\mathrm{MO}(f)$ is used to obtain the necessary and sufficient conditions for the boundedness and compactness of the Hankel operator or multiplication operator $[14,15]$. This work is partially motivated by using Berezin transform $\tilde{f}$ to obtain necessary and sufficient conditions for the compactness of multiplication operator on the weighted Bergman space of the unit ball.

Throughout the paper, we will use the letter $c$ to denote a generic positive constant that can change its value at each occurrence.

\section{Main Results}

In this section, we give the necessary and sufficient conditions for the compactness of multiplication operator on the weighted Bergman space of the unit ball. We furthermore obtain the necessary and sufficient conditions for the compactness of Toeplitz operator and Hankel operator.

Theorem 1. Suppose $|f| /(1-|z|)^{4 n+4 \alpha+4}$ is bounded on $B_{n}$. Then $M_{f}$ is compact operator on $A_{\alpha}^{2}\left(B_{n}\right)$ if and only if $\widetilde{|f|}(z) \rightarrow 0$ as $z \rightarrow \partial B_{n}$.

Proof. Suppose $\widetilde{|f|}(z) \rightarrow 0$ as $z \rightarrow \partial B_{n}$. Since

$$
\left\langle M_{f} g, h\right\rangle_{\alpha}=\langle f g, h\rangle_{\alpha}=\langle g, \bar{f} h\rangle_{\alpha},
$$

it is clear that $\left(M_{f}\right)^{*}=M_{\bar{f}}$. It suffices to prove that the operator $\left(M_{f}\right)^{*}$ is compact by showing that $\left(M_{f}\right)^{*}$ can be approximated by compact operators in the operator norm.

Let $g \in L^{2}\left(B_{n}, d v_{\alpha}\right)$. Then $\left(M_{f}\right)^{*} g \in A_{\alpha}^{2}\left(B_{n}\right)$, so we have

$$
\begin{aligned}
\left(\left(M_{f}\right)^{*} g\right)(z) & =\left\langle\left(M_{f}\right)^{*} g, K_{z}^{\alpha}\right\rangle_{\alpha} \\
& =\int_{B_{n}} g(w) \overline{f(w) K_{z}^{\alpha}(w)} d v_{\alpha}(w),
\end{aligned}
$$

for $z \in B_{n}$.
We define for $0<r<1$ an operator $S_{r}$ by

$$
\left(S_{r} g\right)(z)=\int_{B_{n}} \chi_{r B_{n}}(z) g(w) \overline{f(w) K_{z}^{\alpha}(w)} d v_{\alpha}(w)
$$

Since $|f| /(1-|z|)^{4 n+4 \alpha+4}$ is bounded on $B_{n}$, we prove that

$$
\begin{aligned}
\int_{B_{n}} \int_{B_{n}}\left|\chi_{r B_{n}}(z) \overline{f(w) K_{z}^{\alpha}(w)}\right|^{2} d v_{\alpha}(w) d v_{\alpha}(z) \\
\quad=\int_{r B_{n}} \int_{B_{n}}\left|\overline{f(w) K_{z}^{\alpha}(w)}\right|^{2} d v_{\alpha}(w) d v_{\alpha}(z) \\
\quad<+\infty .
\end{aligned}
$$

Thus, the operator $S_{r}$ is a Hilbert-Schmidt operator. Since

$$
\begin{aligned}
& \left(\left(M_{f}\right)^{*}-S_{r}\right) g(z) \\
& \quad=\int_{B_{n}} g(w) \chi_{B_{n} \backslash r B_{n}}(z) \overline{f(w) K_{z}^{\alpha}(w)} d v_{\alpha}(w),
\end{aligned}
$$

$\left(M_{f}\right)^{*}-S_{r}$ is an integral operator with kernel $K_{r}^{f}(z, w)=$ $\chi_{B_{n} \backslash r B_{n}}(z) \overline{f(w) K_{z}^{\alpha}(w)}$.

By Schur's test, whenever there exists a positive measurable function $h$ on $B_{n}$ and constants $c_{1}$ and $c_{2}$ such that

$$
\int_{B_{n}}\left|K_{r}^{f}(z, w)\right| h(z) d v_{\alpha}(z) \leq c_{1} h(w)
$$

for all $w$ in $B_{n}$, and

$$
\int_{B_{n}}\left|K_{r}^{f}(z, w)\right| h(w) d v_{\alpha}(w) \leq c_{2} h(z)
$$

for all $z$ in $B_{n}$, we have

$$
\left\|\left(M_{f}\right)^{*}-S_{r}\right\| \leq c_{1} c_{2} .
$$

Let $h(z)=\left(1-|z|^{2}\right)^{(n+\alpha+1) / 2}$. Since

$$
\begin{gathered}
K_{z}^{\alpha}\left(\psi_{z}(v)\right) k_{z}^{\alpha}(v)=\frac{1}{\left(1-|z|^{2}\right)^{(n+1+\alpha) / 2}}, \\
1-\left|\psi_{z}(v)\right|^{2}=\frac{\left(1-|z|^{2}\right)\left(1-|v|^{2}\right)}{|1-\langle v, z\rangle|^{2}},
\end{gathered}
$$


and Hölder inequality, it is easy to prove that

$$
\begin{aligned}
& \int_{B_{n}} \chi_{B_{n} \backslash r B_{n}}(z)\left|f(w) K_{z}^{\alpha}(w)\right| \frac{\left(1-|w|^{2}\right)^{(n+\alpha+1) / 2}}{\left(1-|z|^{2}\right)^{(n+\alpha+1) / 2}} d v_{\alpha}(w) \\
& =\int_{B_{n}} \chi_{B_{n} \backslash r B_{n}}(z)\left|f(w) k_{z}^{\alpha}(w)\right| \frac{\left(1-|w|^{2}\right)^{(n+\alpha+1) / 2}}{\left(1-|z|^{2}\right)^{n+\alpha+1}} d v_{\alpha}(w) \\
& =\int_{B_{n}} \chi_{B_{n} \backslash r B_{n}}(z)\left|f\left(\psi_{z}(w)\right) k_{z}^{\alpha}\left(\psi_{z}(w)\right)\right| \\
& \times \frac{\left(1-\left|\psi_{z}(w)\right|^{2}\right)^{(n+\alpha+1) / 2}}{\left(1-|z|^{2}\right)^{n+\alpha+1}}\left|k_{z}^{\alpha}(w)\right|^{2} d v_{\alpha}(w) \\
& =\int_{B_{n}} \chi_{B_{n} \backslash r B_{n}}(z)\left|f\left(\psi_{z}(w)\right)\right| \frac{\left(1-|w|^{2}\right)^{(n+\alpha+1) / 2}}{|1-\langle z, w\rangle|^{2 n+2 \alpha+2}} d v_{\alpha}(w) \\
& =\int_{B_{n}} \chi_{B_{n} \backslash r B_{n}}(z) \sqrt{\left|f\left(\psi_{z}(w)\right)\right|} \sqrt{\left|f\left(\psi_{z}(w)\right)\right|} \\
& \times \frac{\left(1-|w|^{2}\right)^{(n+\alpha+1) / 2}}{|1-\langle z, w\rangle|^{2 n+2 \alpha+2}} d v_{\alpha}(w) \\
& \leq \chi_{B_{n} \backslash r B_{n}}(z)\left[\int_{B_{n}}\left|f\left(\psi_{z}(w)\right)\right| d v_{\alpha}(w)\right]^{1 / 2} \\
& \times\left[\int_{B_{n}}\left|f\left(\psi_{z}(w)\right)\right| \frac{\left(1-|w|^{2}\right)^{n+\alpha+1}}{|1-\langle z, w\rangle|^{4 n+4 \alpha+4}} d v_{\alpha}(w)\right]^{1 / 2} \\
& \leq c \chi_{B_{n} \backslash r B_{n}}(z)\left[\int_{B_{n}}\left|f\left(\psi_{z}(w)\right)\right| d v_{\alpha}(w)\right]^{1 / 2} \\
& \times\left[\int_{B_{n}}\left|f\left(\psi_{z}(w)\right)\right| \frac{1}{(1-|z|)^{3 n+3 \alpha+3}} d v_{\alpha}(w)\right]^{1 / 2} \\
& \leq c[\widetilde{|f|}(z)]^{1 / 2} \\
& =c_{1} \text {, }
\end{aligned}
$$

where $c_{1}=c[\widetilde{|f|}(z)]^{1 / 2}, r<|z|<1$.

Since

$$
\begin{gathered}
K_{z}^{\alpha}\left(\psi_{z}(v)\right) k_{z}^{\alpha}(v)=\frac{1}{\left(1-|z|^{2}\right)^{(n+1+\alpha) / 2}} \\
1-\left\langle\psi_{z}(w), z\right\rangle=\frac{1-|z|^{2}}{1-\langle w, z\rangle}
\end{gathered}
$$

then we obtain

$$
\begin{aligned}
& \int_{B_{n}} \chi_{B_{n} \backslash r B_{n}}(z)\left|f(w) K_{z}^{\alpha}(w)\right| \frac{\left(1-|z|^{2}\right)^{(n+\alpha+1) / 2}}{\left(1-|w|^{2}\right)^{(n+\alpha+1) / 2}} d v_{\alpha}(z) \\
& =\int_{B_{n}} \chi_{B_{n} \backslash r B_{n}}(w)\left|f(z) K_{z}^{\alpha}(w)\right| \frac{\left(1-|w|^{2}\right)^{(n+\alpha+1) / 2}}{\left(1-|z|^{2}\right)^{(n+\alpha+1) / 2}} d v_{\alpha}(w) \\
& =\int_{B_{n}} \chi_{B_{n} \backslash r B_{n}}\left(\psi_{z}(w)\right)\left|f(z) K_{z}^{\alpha}\left(\psi_{z}(w)\right)\right| \\
& \quad \times \frac{\left(1-\left|\psi_{z}(w)\right|^{2}\right)^{(n+\alpha+1) / 2}}{\left(1-|z|^{2}\right)^{(n+\alpha+1) / 2}\left|k_{z}^{\alpha}(w)\right|^{2} d v_{\alpha}(w)} \\
& =\int_{B_{n}} \chi_{B_{n} \backslash r B_{n}}\left(\psi_{z}(w)\right)|f(z)| \\
& \times c_{2}, \\
& \times \frac{\left(1-\left|\psi_{z}(w)\right|^{2}\right)^{(n+\alpha+1) / 2}}{|1-\langle z, w\rangle|^{4 n+4 \alpha+4}} d v_{\alpha}(w)
\end{aligned}
$$

where $c_{2}$ is positive number.

By the above analysis, we get (12) and (13). By Schur's test we get $\left\|\left(M_{f}\right)^{*}-S_{r}\right\| \leq c_{1} c_{2}$, where $c_{1} \rightarrow 0$ as $|z| \rightarrow \partial B_{n}$ and $c_{2}$ does not depend on $r$. So $\widetilde{|f|}(z) \rightarrow 0$ as $z \rightarrow \partial B_{n}$ implies that $M_{f}$ is compact on $A_{\alpha}^{2}\left(B_{n}\right)$.

Suppose $M_{f}$ is compact on $A_{\alpha}^{2}\left(B_{n}\right)$.

Since the kernels $k_{z}^{\alpha}$ converge weakly to zero as $z$ tends $\partial B_{n}$, then we have $\left\|M_{f} k_{z}^{\alpha}\right\|_{\alpha, 2}$ converges to zero as $z$ tends $\partial B_{n}$. So we obtain

$$
\widetilde{|f|}(z) \leq\left[\widetilde{|f|^{2}}(z)\right]^{1 / 2}=\left\|M_{f} k_{z}^{\alpha}\right\|_{\alpha, 2} \longrightarrow 0,
$$

as $z \rightarrow \partial B_{n}$.

Let $f \in L^{1}\left(B_{n}, d v_{\alpha}\right)$ and let $p \geq 1$; we say that $f \in$ $\mathrm{BMO}_{\alpha}^{p}\left(B_{n}\right)$ whenever

$$
\|f\|_{\mathrm{BMO}_{\alpha}^{p}}=\sup _{z \in B_{n}}\left\|f \circ \psi_{z}-\tilde{f}(z)\right\|_{\alpha, p}<\infty
$$

Note that $\|\cdot\|_{\mathrm{BMO}_{\alpha}^{p}}$ does not distinguish constants, while $\left|\|f\|_{\alpha, p}=\|f\|_{\mathrm{BMO}_{\alpha}^{p}}+\right| \tilde{f}(0) \mid$ is a norm in $\mathrm{BMO}_{\alpha}^{p}\left(B_{n}\right)$. By Theorem 5 in [16], we know that $\mathrm{BMO}_{\alpha}^{p}\left(B_{n}\right)$ is equivalent to $\mathrm{BMO}_{\partial}^{p}$ (see the definition in [16]).

For any $p \geq 1$, let $\mathrm{VMO}_{\alpha}^{p}$ denote the subspace of $\mathrm{BMO}_{\alpha}^{p}$ consisting of functions $f$ such that

$$
\lim _{|z| \rightarrow 1^{-}}\left\|f \circ \psi_{z}-\tilde{f}(z)\right\|_{\alpha, p}=0
$$

Theorem 2. Suppose $f \in V M O_{\alpha}^{1}$ and $|f| /(1-|z|)^{4 n+4 \alpha+4}$ is bounded on $B_{n}$. Then the following are equivalent: 
(a) $\tilde{f}(z) \rightarrow 0$ as $z \rightarrow \partial B_{n}$;

(b) $M_{f}$ is compact operator on $A_{\alpha}^{2}\left(B_{n}\right)$;

(c) $T_{f}$ is compact operator on $A_{\alpha}^{2}\left(B_{n}\right)$.

Proof. It suffices to prove that $(a) \Leftrightarrow(b)$ and $(a) \Leftrightarrow(c)$.

$(a) \Leftrightarrow(b)$. Since

$$
\begin{aligned}
& |\tilde{f}(z)| \leq \widetilde{f f}(z), \\
& =\int_{B_{n}}(|f(w)|-|\tilde{f}(z)|) \frac{\left(1-|z|^{2}\right)^{n+\alpha+1}}{|1-\langle z, w\rangle|^{2 n+2 \alpha+2}} d v_{\alpha}(w) \\
& \leq \int_{B_{n}}|f(w)-\tilde{f}(z)| \frac{\left(1-|z|^{2}\right)^{n+\alpha+1}}{|1-\langle z, w\rangle|^{2 n+2 \alpha+2}} d v_{\alpha}(w) \\
& =\int_{B_{n}}\left|f \circ \psi_{z}(w)-\tilde{f}(z)\right| d v_{\alpha}(w) \\
& =\left\|f \circ \psi_{z}-\tilde{f}(z)\right\|_{\alpha, 1},
\end{aligned}
$$

then we obtain that $\tilde{f}(z) \rightarrow 0$ as $z \rightarrow \partial B_{n}$ if and only if $\widetilde{|f|}(z) \rightarrow 0$ as $z \rightarrow \partial B_{n}$. By Theorem 1, we obtain that $M_{f}$ is compact operator on $A_{\alpha}^{2}\left(B_{n}\right)$ if and only if $\widetilde{f}(z) \rightarrow 0$ as $z \rightarrow \partial B_{n}$.

$(a) \Leftrightarrow(c)$. It is clear that $\tilde{f}(z) \rightarrow 0$ as $z \rightarrow \partial B_{n}$ if and only if $T_{f}$ with $\mathrm{BMO}_{\alpha}^{1}$ symbol is compact operator on $A_{\alpha}^{2}\left(B_{n}\right)$ in [12]. Since $\mathrm{VMO}_{\alpha}^{1} \subset \mathrm{BMO}_{\alpha}^{1}$, then it is clear that $\tilde{f}(z) \rightarrow 0$ as $z \rightarrow \partial B_{n}$ if and only if $T_{f}$ is compact operator on $A_{\alpha}^{2}\left(B_{n}\right)$.

Corollary 3. Suppose $f \in V M O_{\alpha}^{1},|f| /(1-|z|)^{4 n+4 \alpha+4}$ is bounded on $B_{n}$, and $H_{f}$ is compact operator on $A_{\alpha}^{2}\left(B_{n}\right)$. Then $M_{f-\tilde{f}}$ is compact operator on $A_{\alpha}^{2}\left(B_{n}\right)$.

Proof. Suppose $H_{f}$ is compact operator on $A_{\alpha}^{2}\left(B_{n}\right)$. So we obtain $H_{f-\tilde{f}}$ is compact operator on $A_{\alpha}^{2}\left(B_{n}\right)$. Since $\widetilde{f-\widetilde{f}}(z) \rightarrow 0$ as $z \rightarrow \partial B_{n}$, then $T_{f-\tilde{f}}$ is compact operator on $A_{\alpha}^{2}\left(B_{n}\right)$. Since

$$
M_{f-\tilde{f}} \mathcal{g}=T_{f-\tilde{f}} \mathcal{g}+H_{f-\tilde{f}} \mathcal{g},
$$

we obtain $M_{f-\tilde{f}}$ is compact operator on $A_{\alpha}^{2}\left(B_{n}\right)$.

By Lemma 17 and Theorem 19 in [16] and Theorem 2, we obtain the following theorem.

Theorem 4. Suppose $f \in V M O_{\alpha}^{1}, \tilde{f}(0)=0$, and $|f| /(1-$ $|z|)^{4 n+4 \alpha+4}$ is bounded on $B_{n}$. Then the following are equivalent:
(a) $M_{f}$ is compact operator on $A_{\alpha}^{2}\left(B_{n}\right)$;
(b) $T_{f}$ is compact operator on $A_{\alpha}^{2}\left(B_{n}\right)$;
(c) $H_{f}$ is compact operator on $A_{\alpha}^{2}\left(B_{n}\right)$.

\section{Conflict of Interests}

The authors declare that there is no conflict of interests regarding the publication of this paper.

\section{Acknowledgment}

Kan Zhang is partly supported by NSFC (no. 113711821).

\section{References}

[1] X. Wang, G. Cao, and J. Xia, "Toeplitz operators on FockSobolev spaces with positive measure symbols," Science China Mathematics, vol. 57, no. 7, pp. 1443-1462, 2014.

[2] K. Hedayatian and L. Karimi, "On convexity of composition and multiplication operators on weighted Hardy spaces," Abstract and Applied Analysis, vol. 2009, Article ID 931020, 9 pages, 2009.

[3] P. S. Bourdon, "Fredholm multiplication and composition operators on the Hardy space," Integral Equations and Operator Theory, vol. 13, no. 4, pp. 607-610, 1990.

[4] K. Stroethoff and D. C. Zheng, "Toeplitz and Hankel operators on Bergman spaces," Transactions of the American Mathematical Society, vol. 329, no. 2, pp. 773-794, 1992.

[5] K. H. Zhu, "Multipliers of BMO in the Bergman metric with applications to Toeplitz operators," Journal of Functional Analysis, vol. 87, no. 1, pp. 31-50, 1989.

[6] K. H. Zhu, Spaces of Holomorphic Functions in the Unit Ball, Springer, New York, NY, USA, 2005.

[7] B. Yousefi, "Unicellularity of the multiplication operator on Banach spaces of formal power series," Studia Mathematica, vol. 147, no. 3, pp. 201-209, 2001.

[8] B. S. Komal and S. Gupta, "Multiplication operators between Orlicz spaces," Integral Equations and Operator Theory, vol. 41, no. 3, pp. 324-330, 2001.

[9] R. K. Singh and J. S. Manhas, "Multiplication operators on weighted spaces of vector-valued continuous functions," Journal of the Australian Mathematical Society (Series A), vol. 50, no. 1, pp. 98-107, 1991.

[10] R. Yoneda, "Multiplication operators, integration operators and companion operators on weighted Bloch space," Hokkaido Mathematical Journal, vol. 34, no. 1, pp. 135-147, 2005.

[11] R. F. Allen and F. Colonna, "Isometries and spectra of multiplication operators on the Bloch space," Bulletin of the Australian Mathematical Society, vol. 79, no. 1, pp. 147-160, 2009.

[12] K. Zhang, C. M. Liu, and Y. F. Lu, "Toeplitz operators with BMO symbols on the weighted Bergman space of the unit ball," Acta Mathematica Sinica (English Series), vol. 27, no. 11, pp. 21292142, 2011.

[13] A. Dieudonne and E. Tchoundja, "Toeplitz operators with $L^{1}$ symbols on Bergman spaces in the unit ball of $C^{n}$," Advances in Pure and Applied Mathematics, vol. 2, no. 1, pp. 65-88, 2011.

[14] M. Jovovic and D. Zheng, "Compact operators and Toeplitz algebras on multiply-connected domains," Journal of Functional Analysis, vol. 261, no. 1, pp. 25-50, 2011.

[15] J. L. Wang and Z. Wu, "Multipliers between BMO spaces on open unit ball," Integral Equations and Operator Theory, vol. 45, no. 2, pp. 231-249, 2003.

[16] K. H. Zhu, "BMO and Hankel operators on Bergman spaces," Pacific Journal of Mathematics, vol. 155, no. 2, pp. 377-395, 1992. 


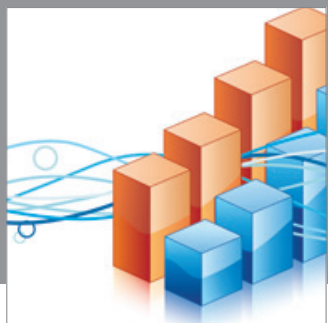

Advances in

Operations Research

mansans

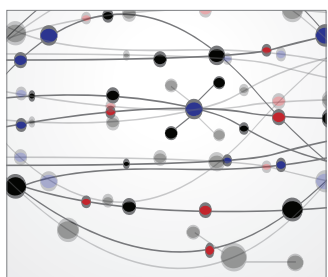

The Scientific World Journal
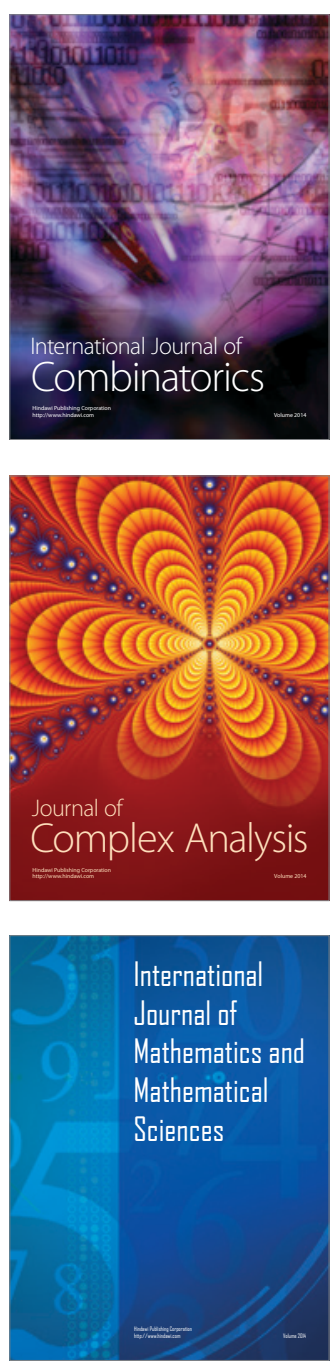
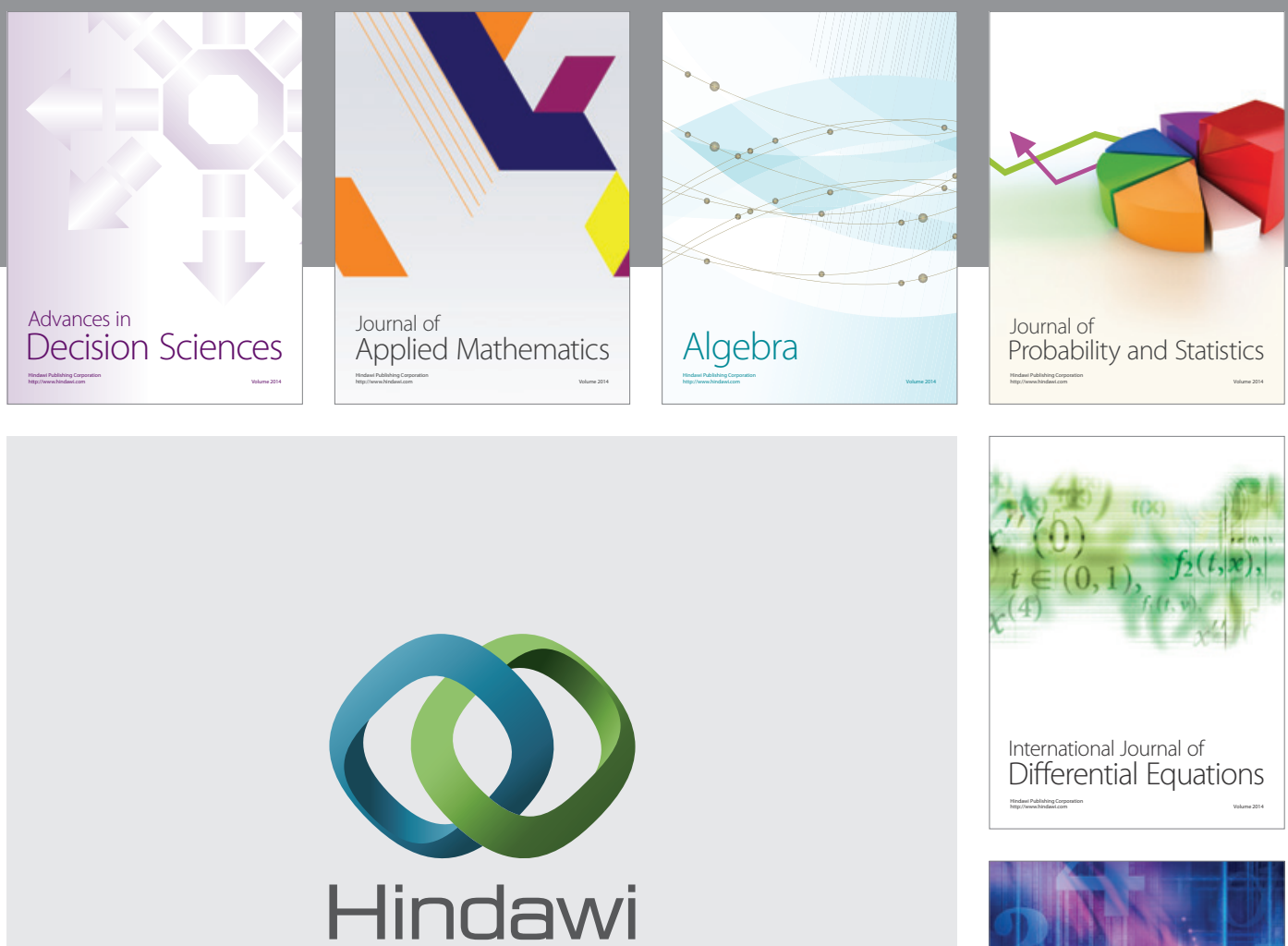

Submit your manuscripts at http://www.hindawi.com
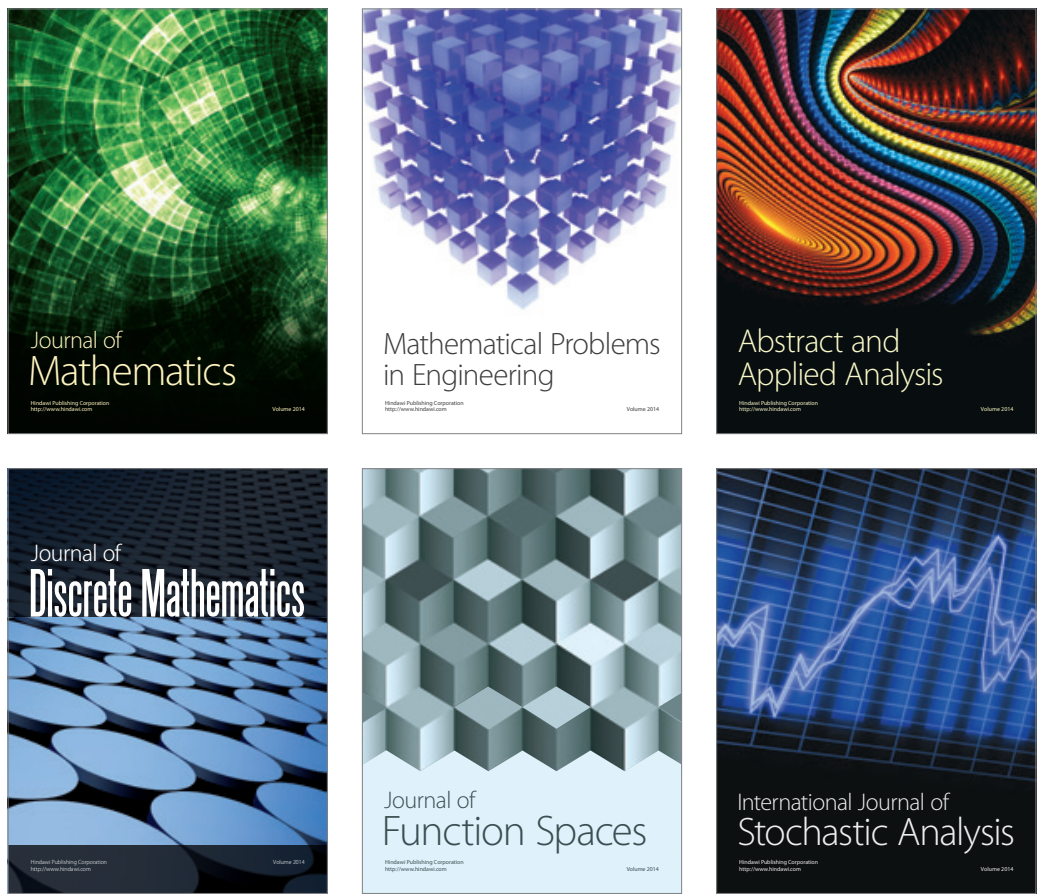

Journal of

Function Spaces

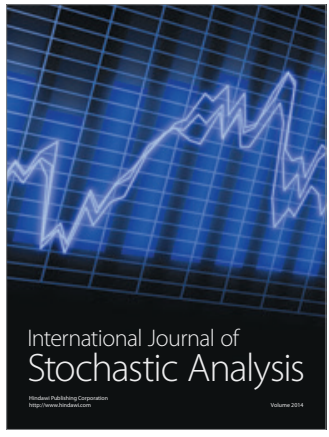

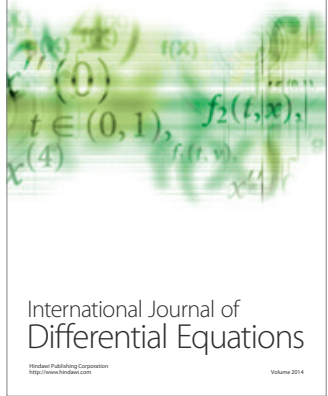
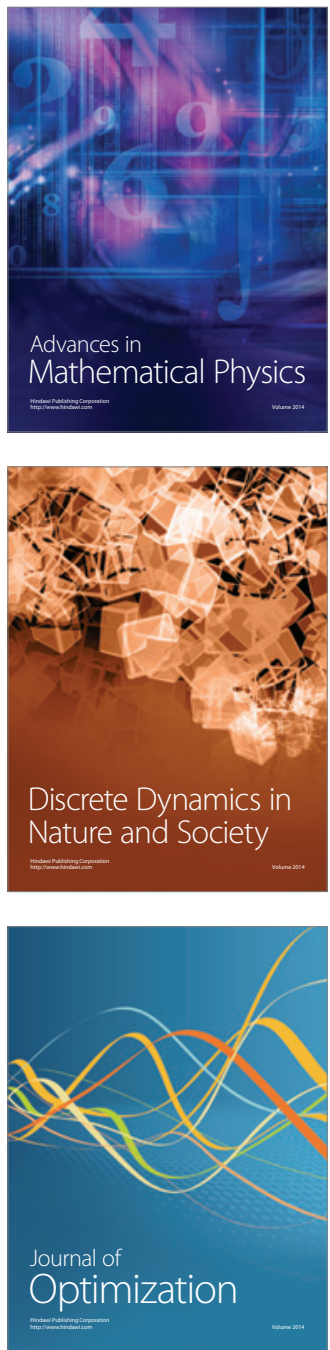